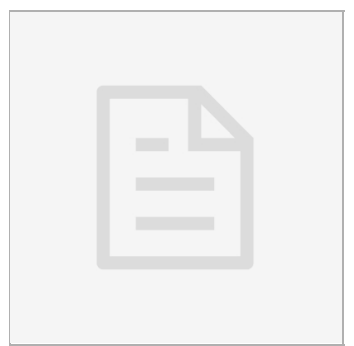

SEP 20, 2017

OPEN OACCESS

DOI:

dx.doi.org/10.17504/protocol s.io.imqce5w

Protocol Citation: ChaoYuan Yeh 2017. Analysis of choroid thickness on OCT image using MATLAB.

protocols.io

https://dx.doi.org/10.17504/p rotocols.io.imqcc5w

License: This is an open access protocol distributed under the terms of the Creative Commons Attribution License, which permits unrestricted use, distribution, and reproduction in any medium, provided the original author and source are credited

Protocol status: Working

Created: Jun 26, 2017

Last Modified: Mar 28,

2018

PROTOCOL integer ID:

6544

\section{(7) Analysis of choroid thickness on OCT image using MATLAB}

\section{Chao-Yuan}

Yeh $^{1}$

${ }^{1}$ University of Southern California

Chao-Yuan Yeh

\section{ABSTRACT}

This protocol describes how to use a custom written MATLAB script to analyze thickness of choroid on OCT images of both eyes.

1 Make sure you have MATLAB 2016b (or newer) with image processing toolbox installed on your computer.

2 Dowload the MATLAB scripts from https://github.com/joe-of-all-trades/OCT_Analysis

3 On the Optovue OCT machine, export cross line OCT images. To allow inperpolation, four cross line images are required for each eye. Single cross line image can still be processed by OCT_ImageAnalysis_for_CrossLine.m.

4 Run $\boldsymbol{O C T}$ _file_processing. $\boldsymbol{m}$ to process OCT images. This script works on raw image generated by Optovue OCT machines. It will make the file name easier to handle and extract image content from cross line scans and line scans and save them into tiff format.

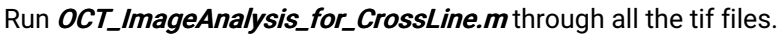




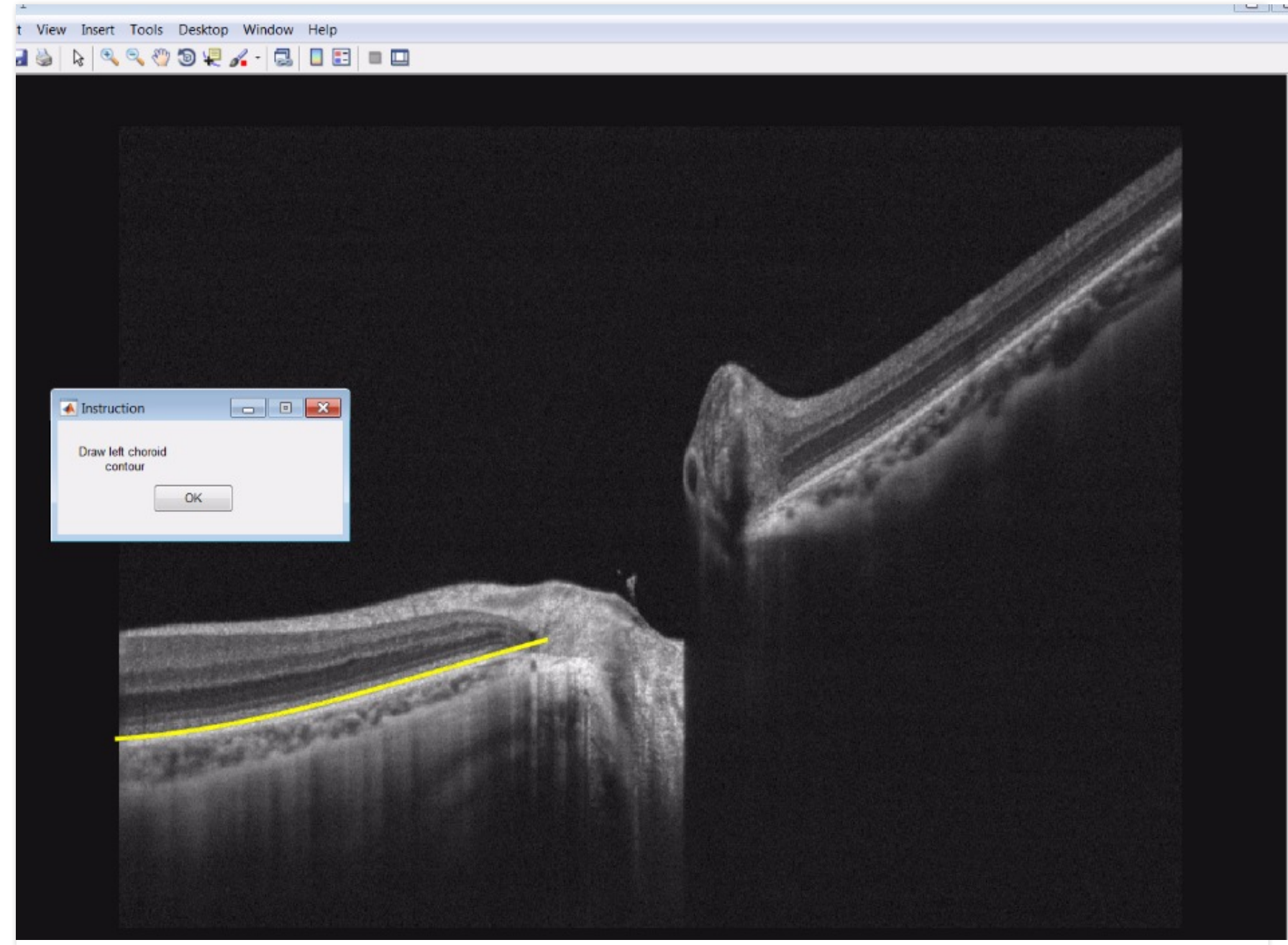

Then follow the prompt and draw along the contour of the choroid on the left side of the image. 


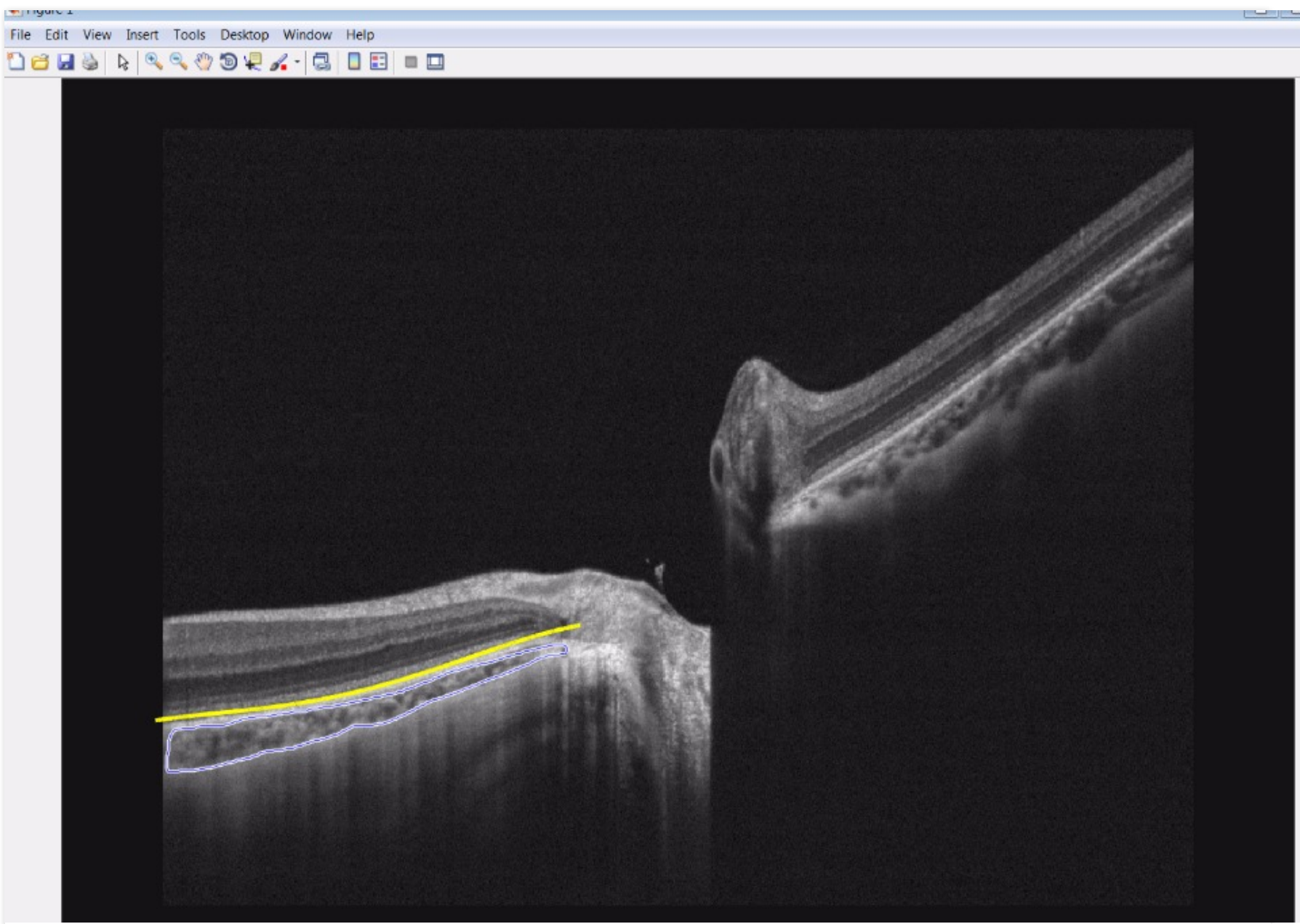

After you're done, choroid thickness will be calculated for you. Red lines represent auxiliary lines perpendicular to the curve. Green lines represent calculated thickness of the curve. 


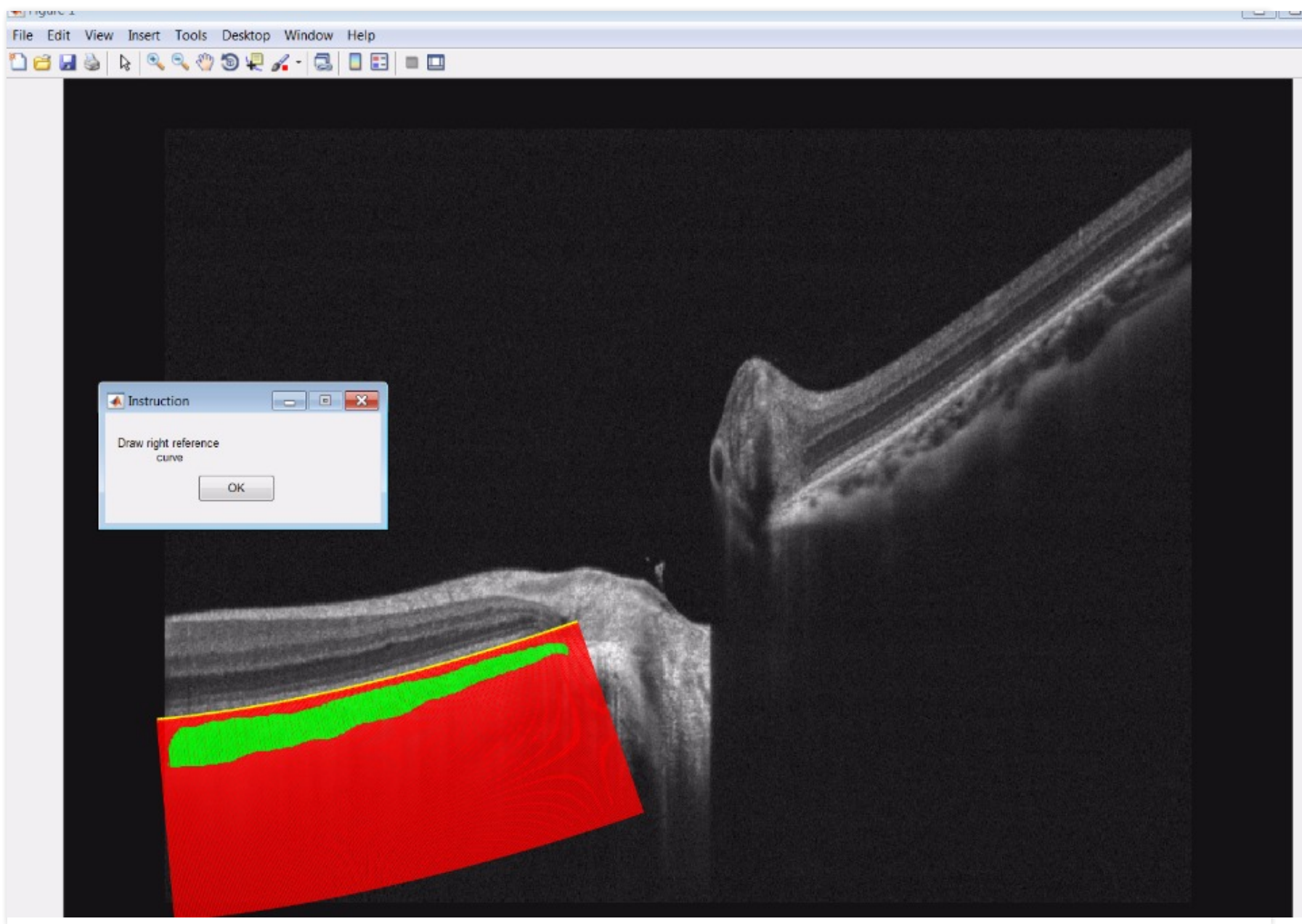

Repeat this process for the right side of the image. Another figure will appear and summarize the thickness measurement. 


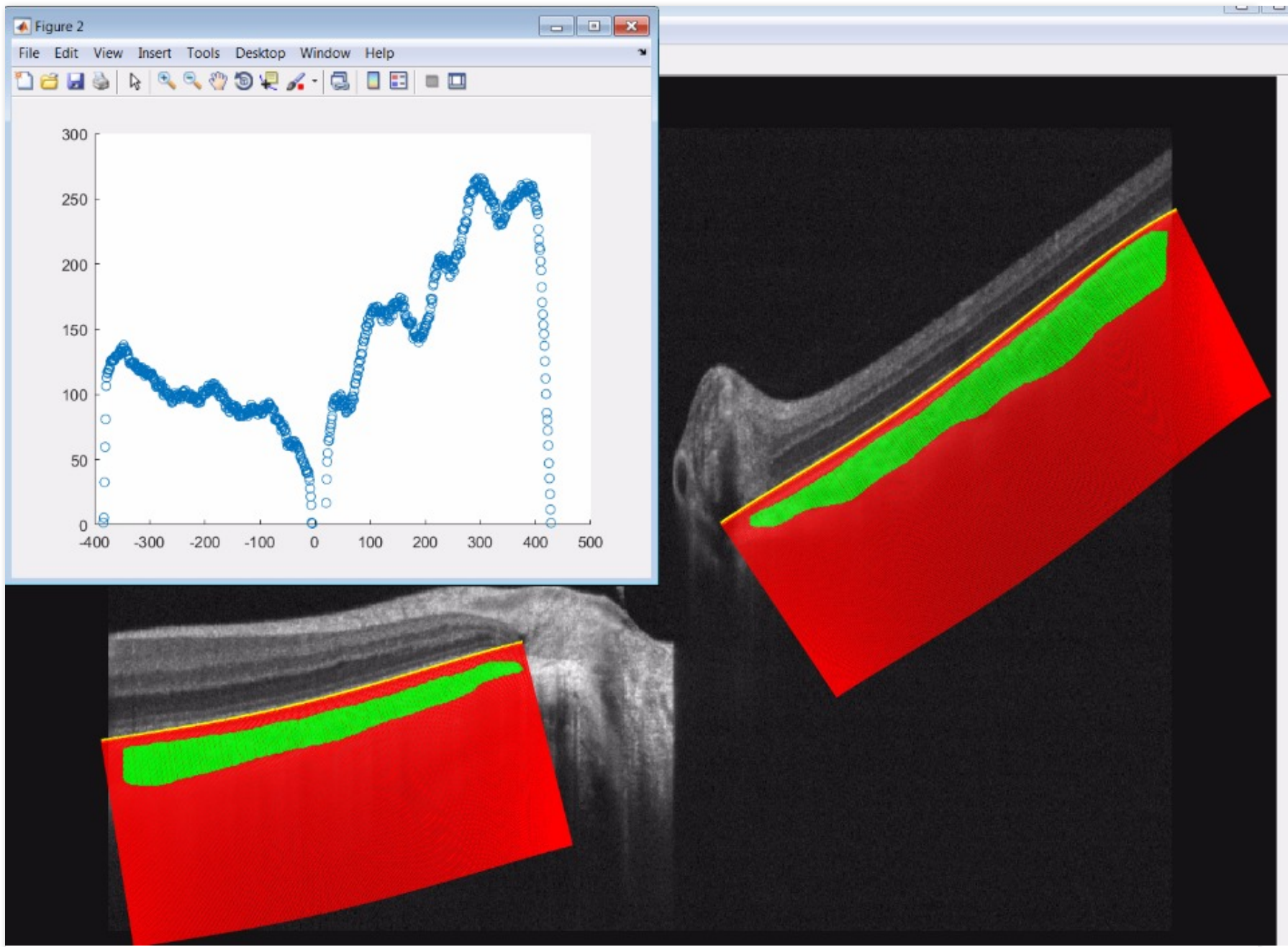

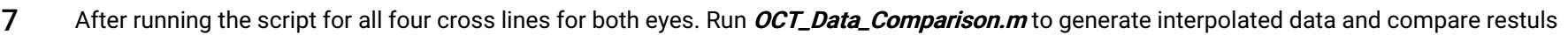
between two eyes. Choroid fhickness data in eight corners will be plotted and an interpolated picture will be shown. An xls file summarizing statistics will also be generated. 


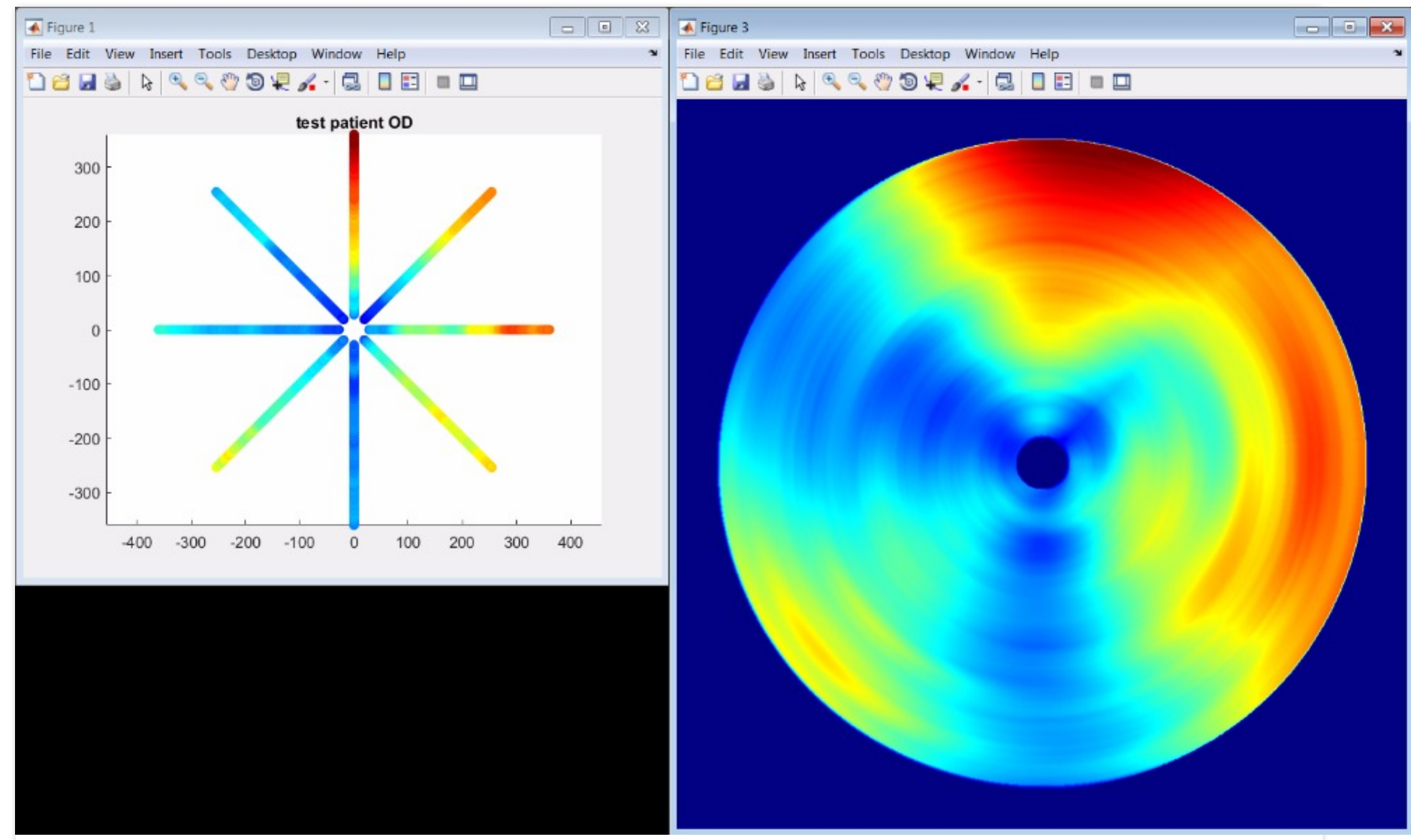

